\title{
THE MINIMUM SIZE OF A GRAPH WITH GIVEN TREE CONNECTIVITY
}

\author{
YUEFANG SUN \\ Department of Mathematics \\ Shaoxing University \\ Zhejiang 312000, P.R. China \\ e-mail: yuefangsun2013@163.com \\ Bin SHENG ${ }^{1}$ \\ College of Computer Science and Technology \\ Nanjing University of Aeronautics and Astronautics \\ Jiangsu 211100, China \\ e-mail: shengbinhello@nuaa.edu.cn
}

AND

\section{ZEMIN JIN}

Department of Mathematics

Zhejiang Normal University

Zhejiang 321004, P.R. China

e-mail: zeminjin@zjnu.cn

\begin{abstract}
For a graph $G=(V, E)$ and a set $S \subseteq V$ of at least two vertices, an $S$-tree is a such subgraph $T$ of $G$ that is a tree with $S \subseteq V(T)$. Two $S$ trees $T_{1}$ and $T_{2}$ are said to be internally disjoint if $E\left(T_{1}\right) \cap E\left(T_{2}\right)=\emptyset$ and $V\left(T_{1}\right) \cap V\left(T_{2}\right)=S$, and edge-disjoint if $E\left(T_{1}\right) \cap E\left(T_{2}\right)=\emptyset$. The generalized local connectivity $\kappa_{G}(S)$ (generalized local edge-connectivity $\lambda_{G}(S)$, respectively) is the maximum number of internally disjoint (edge-disjoint, respectively) $S$-trees in $G$. For an integer $k$ with $2 \leq k \leq n$, the generalized $k$-connectivity (generalized $k$-edge-connectivity, respectively) is defined as $\kappa_{k}(G)=\min \left\{\kappa_{G}(S)|S \subseteq V(G)| S \mid,=k\right\} \quad\left(\lambda_{k}(G)=\min \left\{\lambda_{G}(S) \mid S \subseteq\right.\right.$ $V(G),|S|=k\}$, respectively).
\end{abstract}

\footnotetext{
${ }^{1}$ Corresponding author.
} 
Let $f(n, k, t)(g(n, k, t)$, respectively) be the minimum size of a connected graph $G$ with order $n$ and $\kappa_{k}(G)=t\left(\lambda_{k}(G)=t\right.$, respectively), where $3 \leq k \leq n$ and $1 \leq t \leq n-\left\lceil\frac{k}{2}\right\rceil$. For general $k$ and $t, \mathrm{Li}$ and Mao obtained a lower bound for $g(n, k, t)$ which is tight for the case $k=3$. We show that the bound also holds for $f(n, k, t)$ and is tight for the case $k=3$. When $t$ is general, we obtain upper bounds of both $f(n, k, t)$ and $g(n, k, t)$ for $k \in\{3,4,5\}$, and all of these bounds can be attained. When $k$ is general, we get an upper bound of $g(n, k, t)$ for $t \in\{1,2,3,4\}$ and an upper bound of $f(n, k, t)$ for $t \in\{1,2,3\}$. Moreover, both bounds can be attained.

Keywords: generalized connectivity, tree connectivity, generalized $k$-connectivity, generalized $k$-edge-connectivity, packing.

2010 Mathematics Subject Classification: 05C05, 05C35, 05C40, 05C70.

\section{REFERENCES}

[1] J.A. Bondy and U.S.R. Murty, Graph Theory (Springer, Berlin, 2008).

[2] G. Chartrand, F. Okamoto and P. Zhang, Rainbow trees in graphs and generalized connectivity, Networks 55 (2010) 360-367. doi:10.1002/net.20339

[3] L. Chen, X. Li, M. Liu and Y. Mao, A solution to a conjecture on the generalized connectivity of graphs, J. Comb. Optim. 33 (2017) 275-282. doi:10.1007/s10878-015-9955-x

[4] X. Cheng and D. Du, Steiner Trees in Industry (Dordrecht, Kluwer Academic Publisher, 2001).

[5] D. Cieslik, Steiner Minimal Trees (Nonconvex Optimization and Its Applications, Springer, 1998).

[6] D. Du and X. Hu, Steiner Tree Problems in Computer Communication Networks (World Scientific, 2008).

[7] M. Grötschel, A. Martin and R. Weismantel, Packing Steiner trees: A cutting plane algorithm and commputational results, Math. Program. 72 (1996) 125-145. doi:10.1007/BF02592086

[8] M. Grötschel, A. Martin and R. Weismantel, The Steiner tree packing problem in VLSI design, Math. Program. 78 (1997) 265-281. doi:10.1007/BF02614374

[9] M. Hager, Pendant tree-connectivity, J. Combin. Theory Ser. B 38 (1985) 179-189. doi:10.1016/0095-8956(85)90083-8

[10] H. Li, X. Li, Y. Mao and Y. Sun, Note on the generalized connectivity, Ars Combin. 114 (2014) 193-202.

[11] H. Li, X. Li, Y. Mao and J. Yue, Note on the spanning-tree packing number of lexicographic product graphs, Discrete Math. 338 (2015) 669-673. doi:10.1016/j.disc.2014.12.007 
[12] H. Li, X. Li and Y. Sun, The generalized 3-connectivity of Cartesian product graphs, Discrete Math. Theor. Comput. Sci. 14 (2012) 43-54.

doi:10.1016/j.commatsci.2011.09.003

[13] S. Li, Some Topics on Generalized Connectivity of Graphs, PhD Thesis (Nankai University, 2012).

[14] X. Li and Y. Mao, A survey on the generalized connectivity of graphs. arXiv:1207.1838[math.CO]

[15] X. Li and Y. Mao, The generalized 3-connectivity of lexicographic product graphs, Discrete Math. Theor. Comput. Sci. 16 (2014) 339-354. doi:10.1007/978-3-319-12691-3_31

[16] X. Li and Y. Mao, Nordhaus-Gaddum-type results for the generalized edgeconnectivity of graphs, Discrete Appl. Math. 185 (2015) 102-112. doi:10.1016/j.dam.2014.12.009

[17] X. Li and Y. Mao, The minimal size of a graph with given generalized 3-edgeconnectivity, Ars Combin. 118 (2015) 63-72.

[18] X. Li and Y. Mao, Graphs with large generalized (edge)-connectivity, Discuss. Math. Graph Theory 36 (2016) 931-958.

doi:10.7151/dmgt.1907

[19] X. Li and Y. Mao, Generalized Connectivity of Graphs (SpringerBriefs in Mathematics, Springer, Switzerland, 2016).

[20] X. Li, Y. Mao and Y. Sun, On the generalized (edge)-connectivity of graphs, Australas. J. Combin. 58 (2014) 304-319.

[21] Y. Mao, Steiner distance in graphs-a survey. arXiv:1708.05779.[math.CO]

[22] C.St.J.A. Nash-Williams, Edge-disjonint spanning trees of finite graphs, J. London Math. Soc. 36 (1961) 445-450. doi:10.1112/jlms/s1-36.1.445

[23] K. Ozeki and T. Yamashita, Spanning trees: a survey, Graphs Combin. 27 (2011) $1-26$. doi:10.1007/s00373-010-0973-2

[24] E. Palmer, On the spanning tree packing number of a graph: a survey, Discrete Math. 230 (2001) 13-21. doi:10.1016/S0012-365X(00)00066-2

[25] Y. Sun, Generalized 3-edge-connectivity of Cartesian product graphs, Czechoslovak Math. J. 65 (2015) 107-117. doi:10.1007/s10587-015-0162-9

[26] Y. Sun, Sharp upper bounds for generalized edge-connectivity of product graphs, Discuss. Math. Graph Theory 36 (2016) 833-843. doi:10.7151/dmgt.1924 
[27] Y. Sun, A sharp lower bound for the generalized 3-edge-connectivity of strong product graphs, Discuss. Math. Graph Theory 37 (2017) 975-988. doi:10.7151/dmgt.1982

[28] Y. Sun and X. Li, On the difference of two generalized connectivities of a graph, J. Comb. Optim. 33 (2017) 283-291. doi:10.1007/s10878-015-9956-9

[29] Y. Sun and S. Zhou, Tree connectivities of Cayley graphs on Abelian groups with small degrees, Bull. Malays. Math. Sci. Soc. 39 (2016) 1673-1685. doi:10.1007/s40840-015-0147-8

[30] W. Tutte, On the problem of decomposing a graph into $n$ connected factors, J. Lond. Math. Soc. 36 (1961) 221-230. doi:10.1112/jlms/s1-36.1.221

Received 16 October 2017 Revised 28 November 2018 Accepted 28 November 2018 